\title{
Wachstumshormon und extrarenale Elektrolytverteilung
}

\author{
Von Th. GüNTher und C. Goecke \\ Aus dent Pbysiologisch-cbemischen Institut der Freien Universität Berlin (Direktor: Prof. Dr. Dr. E. Scbütte)
}

(Eingegangen am 25. Juli 1966)

\begin{abstract}
An Ratten wurde nach Zufuhr von STH der Rohrzuckerverteilungstaum (EZF) sowie der Natrium- und Kalium-Gehalt in Leber und Muskulatur untersucht. Unter STH penetriert Rohrzucker in die Leberzellen; in der Muskulatur erfolgt eine Vermehrung der EZF. Der Kalium-Bestand in Leber und Muskulatur vergrößert sich. Die intrazelluläre Kalium-Konzentration in Leber- und Muskelzellen nimmt dabei zu, die intrazelluläre Natrium-Konzentration nimmt ab. Aus der Aktivierung des Kalium-, Aminosäure- und Zucker-Transportes durch STH wird gefolgert, daß im tierischen Organismus eine Beziehung zwischen Permeabilität, aktivem Transport und Wachstum besteht.
\end{abstract}

The sucrose free space (SFS) and the $\mathrm{Na}$ and $\mathrm{K}$ content of liver and muscle were measured in rats after the administration of STH. As a result of STH treatment, sucrose enters the liver cells, the SFS increases in the muscle, the intracellular liver and muscle potassium levels are increased, and the intracellular sodium is decreased. From the activation of the transport of potassium, amino acids and sugar by STH, it is concluded that permeability, active transport and growth are interrelated in the animal organism.

An Pflanzenzellen wurde nachgewiesen, daß aktiver Transport von Zuckern, Aminosäuren und Phosphat eng mit dem Wachstum gekoppelt ist. Wachsende Pflanzenteile können ihre Substrate aus anderen Pflanzenteilen gegen den Konzentrationsgradienten akkumulieren. Substanzen, die bei Pflanzen Wachstum induzieren, erhöhen in Pflanzen auch den aktiven Transport (1). Diese Beziehung scheint, auch für den tierischen Organismus zuzutreffen. Schnell wachsende Organe haben eine höhere intrazelluläre Aminosäurekonzentration als langsam wachsende (2). Hormone wie ACTH, TSH, FSH, Testosteron oder Östradiol, die das Wachstum eines bestimmten Organs stimulieren, erhöhen auch die Aminosäurekonzentration in diesem Organ (2). Dies gilt insonderheit für das Wachstumshormon der Hypophyse (STH), das den aktiven Transport von Aminosäuren $(3,4,5)$ und Zuckern (6) fördert. Da sich Kalium hinsichtlich des aktiven Transportes wie Aminosäuren verhalten kann $(7,8)$ und da einige Hormone den Transport von Kalium und Aminosäuren in gleicher Weise aktivieren (9), untersuchten wir, ob das Wachstumshormon auch die Verteilung von Kalium, dem typischen intrazellulären Kation, ändert.

\section{Methodik}

Sieben männliche Ratten im Gewicht von 235-270 g (Mittel: $253 \mathrm{~g}$ ) erhieiten 14 Tage lang tägl. je $0,7 \mathrm{mg}$ RinderhypophysenWachstumshormon ${ }^{1}$ ) subcutan injiziert. Es wurde zunächst eine 1-proz. Suspension des Hormons in 0,9 -proz. $\mathrm{NaCl}$ hergestellt. Nach Zugabe von etwa $0,05 \mathrm{~m} l 0,1 \mathrm{~N} \mathrm{NaOH}$ erhält man eine klare

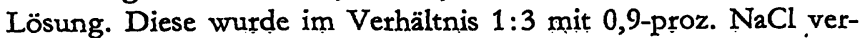
dünnt. Hiervon wurden $0,2 \mathrm{ml}$ tägl. injiziert. Jeden dritten Tag wurde neue Hormonlösung bereitet. Die restliche Lösung wurde bei $4^{\circ}$ aufbewahrt. 2 Stdn. nach der letzten Injektion erhielten die Tiere $1,5 \mathrm{ml}$ einer 14-proz. Rohrzuckerlösung i. v. injiziert. 15 Min. danach wurden sie durch Genickschlag getötet.

Die Aufarbeitung der Gewebe sowie die Bestimmung des Rohrzuckerverteilungsraumes, des Wasser- und Elektrolytgehaltes wurde an anderer Stelle ausführlich beschrieben (10).

Als Kontrollen dienten sechs gleichschwere, unbehandelte Tiere. Alle Tiere erhielten Standardpreßfutter und Leitungswasser.

1) Wir danken Herrn Dr. E. WrLhelmi, Endocrinology Studie Section, National Institute of Health, Bethesda, Md., für die großzügige Uberlassung des gereinigten Rinderhypophysen-Wachstumshormon (NIH-GH-B , $_{3}$.

\section{Ergebnisse}

Während der Zufuhr des Wachstumshormon nahm das Gewicht der behandelten Tiere stärker zu als das Gewicht einer Kontrollgruppe (Abb. 1).

Die Na- und K-Konzentrationen im Serum änderten sich nicht (Tab. 1): In der Leber stieg der Na-Gehalt von 81,2 auf $87,2 \mathrm{mMol} / \mathrm{kg}$ Trockengewicht (TG) und der K-Gehalt von 278,0 auf $293,7 \mathrm{mMol} / \mathrm{kg}$ TG (Tab. 2). Der Rohrzuckerraum (EZF) nahm bei gleichbleibendem

Tab. 1

Natrium- und Kalium-Konzentration im Serum (Werte in mMol ll)

\begin{tabular}{lcc}
\hline Tiergruppe & $\mathrm{Na}$ & $\mathrm{K}$ \\
\hline Unbehandelt & $152,5 \pm 0,9$ & $7,1 \pm 0,1$ \\
STH-Behandlung & $154,2 \pm 2,8$ & $7,5 \pm 0,2$ \\
\hline
\end{tabular}

Wassergehalt von 16,9 auf $24,5 \%$ Frischgewicht (FG) zu. Der Rohrzuckerraum der STH-behandelten Tiere ist also in der Leber mit $24,5 \%$ größer als der Na-Verteilungsraum $(18,4 \%)$. Daraus folgt, daß nach STHGaben Rohrzucker in die Leberzellen gelangt sein muß. Die wahre Größe der EZF in der Leber läßt sich somit nicht angeben. Der Na-Verteilungsraum kann nur näherungsweise als $\mathrm{Ma} B$ der EZF dienen, da man nicht weiß, ob unter STH auch Natrium in die Leberzelle gelangt. Der $\mathrm{Na}$-Verteilungstaum gibt lediglich die obere Grenze der EZF an. Deswegen läßt sich auch der genaue Wert für die intrazelluläre Kalium-Konzentration in der

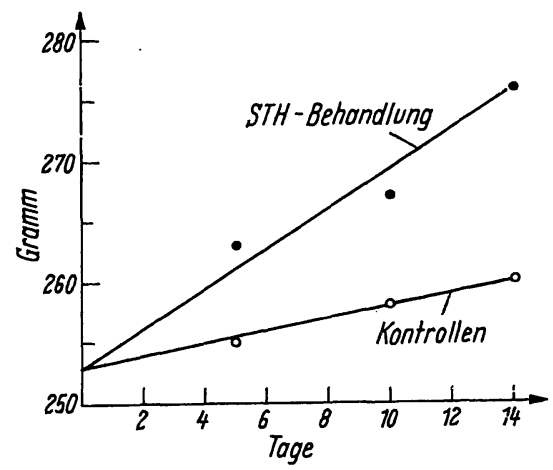

Abb. 1

Mittlere Gewichtzunahme von 7 Ratten, die tägl. $0,7 \mathrm{mg} \mathrm{STH}$ crhielten und 7 unbehandelten Kontrolltieren 
Tab. 2

Analysenwerte der Organe

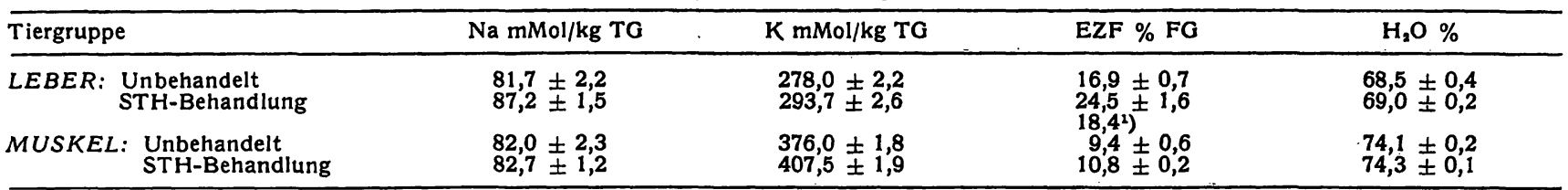

1) Natrium-Verteilungsraum

Tab. 3

Extra- und intrazelluläre Verteilung von Natrium und Kalium

\begin{tabular}{|c|c|c|c|c|c|c|c|c|c|c|}
\hline \multicolumn{2}{|l|}{ Tiergruppe } & \multicolumn{2}{|c|}{$\underset{\mathrm{Na}}{\mathrm{mMol} / \mathrm{kg}} \underset{\mathrm{FG}}{\mathrm{F}}$} & \multicolumn{2}{|c|}{$\begin{array}{l}\text { extrazell. Anteil } \\
\mathrm{mMol} / \mathrm{kg} \mathrm{FG} \\
\mathrm{Na}\end{array}$} & \multicolumn{2}{|c|}{$\begin{array}{l}\text { intrazell. Anteil } \\
\mathrm{mMol} / \mathrm{kg} \mathrm{FG} \\
\mathrm{Na}\end{array}$} & $\underset{\mathrm{ml} / \mathrm{kg} \text { FG }}{\mathrm{IZF}}$ & \multicolumn{2}{|c|}{ intrazell. Konz. } \\
\hline LEBER: & $\begin{array}{l}\text { Unbehandelt } \\
\text { STH Be. andlung }\end{array}$ & $\begin{array}{l}25,8 \\
27,0\end{array}$ & $\begin{array}{l}87,7 \\
90,9\end{array}$ & $\begin{array}{l}24,5 \\
26,85\end{array}$ & $\begin{array}{l}1,14 \\
1,31\end{array}$ & 1,3 & $\begin{array}{l}86,6 \\
89,6\end{array}$ & $\begin{array}{l}516 \\
\left.506^{1}\right)\end{array}$ & 2,5 & $\begin{array}{l}168 \\
178\end{array}$ \\
\hline MUSKEL: & $\begin{array}{l}\text { Unbehandelt } \\
\text { STH Eehandlung }\end{array}$ & $\begin{array}{l}21,2 \\
21,3\end{array}$ & $\begin{array}{r}97,5 \\
105,0\end{array}$ & $\begin{array}{l}13,65 \\
15,8\end{array}$ & $\begin{array}{l}0,64 \\
0,77\end{array}$ & $\begin{array}{l}\mathbf{7 , 5} \\
\mathbf{5 , 5}\end{array}$ & $\begin{array}{r}96,9 \\
104,2\end{array}$ & $\begin{array}{l}647 \\
635\end{array}$ & $\begin{array}{r}11,6 \\
8,7\end{array}$ & $\begin{array}{l}150 \\
165\end{array}$ \\
\hline
\end{tabular}

2) Berechnung der IZF mit Hilfe des Na-Raumes

Leber nach Behandlung mit STH nicht angeben; sie hat jedoch auf jeden Fall zugenommen.

In der Muskulatur blieb nach STH-Zufuhr der NatriumGehalt gleich. Der Kalium-Gehalt nahm von 376,0 auf $407,5 \mathrm{mMol} / \mathrm{kg}$ TG zu. Die EZF vergrößerte sich bei konstantem Wassergehalt von 9,4 auf $10,8 \%$ FG (Tab. 2). Wenn der Rohrzuckerraum in der Muskulatur ein exaktes Maß für die Größe der EZF ist, dann folgt, daß der Kalium-Gehalt und die Kalium-Konzentration in den Muskelzellen angestiegen sind, während der Natrium-Gehalt und die Natrium-Konzentration abgenommen haben (Tab. 3).

\section{Diskussion}

Bei Versuchen mit Hormonen in vivo besteht die Möglichkeit, daß die gemessenen Veränderungen gar nicht durch das zugeführte Hormon hervorgerufen werden, sondern sekundär durch andere vom Körper gebildete Hormone bewirkt werden. Da einige Wirkungen des Wachstumshormons wie die Aktivierung der zellulären Aufnahme von Zuckern, Aminosäuren und Kalium auch durch Insulin bewirkt werden $(11,4,12)$, besteht die Möglichkeit, daß die Wirkungen des STH in vivo sekundär durch Insulin zustande kommen, wie dies für die Permeabilitätssteigerung von Zuckern angenommen wurde (2). Nach STH-Gaben an hypophysektomierte Ratten stieg die Insulin-Aktivität im Plasma aber nicht an (12), und nach Zufuhr von STH an hypophysektomierte Ratten nahm die Menge des an das Diaphragma gebundenen Insulins sogar ab (14). Es ist also anzunehmen, daß die beobachteten Änderungen der Wasserund Elektrolytverteilung tatsächlich durch das Wachstumshormon bedingt sind.
Eine Vermehrung der EZF, die wir in der Leber und Muskulatur fanden, konnten Ikkos und Mitarbeiter (15) auch bei Patienten mit Akromegalie mit Hilfe des InulinVerteilungsraumes nachweisen. Dies erklärt dieNatriumRetention, die in Bilanzuntersuchungen ermittelt wurde (16). Die im Verhältnis zur Stickstoff-Retention erhöhte Kalium-Retention, die in derartigen Versuchen ebenfalls festgestellt wurde (17), können wir mit der geringen, aber signifikanten Erhöhung der intrazellulären KaliumKonzentration in Leber und Muskulatur erklären. Damit übereinstimmend wurde bei Patienten mit Akromegalie eine 8-proz. Erhöhung des Kalium-Gehaltes in der Muskulatur bei gleichbleibendem Wassergehalt gefunden (18).

Das Eindringen von Rohrzucker in die Leberzellen steht in Einklang mit Befunden von MeLHuish und Mitarbeiter (19) an Mitochondrien, deren Membranen ähnliche Eigenschaften wie die Zellmembranen haben (20). Danach fühtt STH an isolierten Lebermitochondrien, die in 0,25M Rohrzuckerlösung ( $\mathrm{pH} 7,4$ ) inkubiert wurden, zu einer starken Schwellung, weil infolge einer Permeabilitätserhöhung Rohrzucker in die Mitochondrien penetriert. STH-Präparationen, die zu einer starken Schwellung führten, waren im Wachstumstest sehr aktiv und umgekehrt: Präparationen, die eine geringe Mitochondrienschwellung bewirkten, waren auch im Wachstumstest wenig wirksam. Nimmt man die Aktivierung des Zucker- und Aminosäure-Transportes durch das Wachstumshormon hinzu, so kann man verallgemeinern, daß auch im tierischen Organismus eine Korrelation $z$ wischen Permeabilität und aktivem Transport einerseits und Wachstum andererseits besteht.

\section{Literatur}

1. Mothes, K., Biochemie des aktiven Transportes, S. 189 , Springer-Verlag, Berlin-Göttingen-Heidelberg (1961). — 2. RIGGs, T. R., G. LETwAK und D. KRITCHEVSKY, Actions of Hormones on Molecular Processes, J. Wiley a. S., New York (1964). - 3. Noald, M. W., T. R. Riggs, L. M. Walker und H. N. Christensen, Science (Washington) 126, 1002 (1957). - 4. Kostro, J. L., J. Hотсн кIss und E. KNoBIL, Science (Washington) 130, 1653 (1959). - 5. Riggs, T. R. und L. M. WALker, J. biol. Chemistry 235, 3603
(1960). - 6. Henderson, M. J., H. E. Morgan und C. R. Park, J. biol. Chemistry 236, 2157 (1961). - 7. Christensen, H. N., T. R. Riggs, H. Fischer und J. M. Palatine, J. biol. Chemistry 198, 1 (1952). - 8. Riggs, T. R., L. M. WALKËR und H. N. Christensen, J. biol. Chemistry 233, 1479 (1958). - 9. Günther, Th. und W. WinkelmanN, Naunyn-Schmiedebergs Arch. exp. Pathol. Pharmakol. 242, 277 (1961). - 10. DulCE, H.-J. und TH. GüNTHER, Naunyn-Schmiedèbergs Arch. exp. Pathol. Pharmakol. 
238, 368 (1960). - 11. KIPNIS, D. M. und C. F. CoRI, J. biol. Chemistry 224, 681 (1957). - 12. Akedo, H. und H. N. ChristenSEN, J. biol. Chemistry 237, 118 (1962). - 13. RANDle, P. J., The hypophyseal growth hormone, nature and actions, S. 413, McGrarv-Hall, New York (1955). - 14. PARK, C. R., D. H. Brown, M. Cornblath, W. H. Daughaday und M. E. Krahi, J. biol. Chemistry 197, 151 (1952). - 15. Ikkos, D., H. LJUNGGREN und
R. Lufr, Acta endocr., K'hvn. 22, 211 (1956). - 16. Ikкos, D., R. Luft und C. A. Gemzell, Acta endocr., K'hvn. 32, 341 (1959). - 17. Biglieri, E. G., C. O. Watuington und P. H. Forsham, J. Clin. Endocr., Springfield 21, 361 (1961). - 18. PALMIERI, G. und D. Ikxos, Acta endocr., K'hvn. 48, 469 (1965). - 19. MeLHuish, A. H. und A. L. Greenbaum, Biochem. J. 78, 392 (1961). 20. Tedeschi, H., J. biophysic. biochem. Cytol. 6, 241 (1959).

\title{
Eine einfache Gelfiltrationsmethode zur routinemäßigen Messung der in vitro-Bindung von markiertem Trijodthyronin an Serumeiweiße
}

\author{
Von R. Gränicher, A. Burger, E. Gfeller und H. Studer \\ Aus den endokrinologischen Laboratorien (Leiter: PD Dr. H. Studer) der Mediqinischen Universitätsklinik, Bern \\ (Direktor: Prof. Dr. F. Wyss)
}

(Eingegangen am 10. Oktober 1966)

\begin{abstract}
Es wird eine vereinfachte Gelfiltrationsmethode zur routinemäßigen Bestimmung der in vitro-Bindung von ${ }^{131}$ I-markiertem Trijodthyronin beschrieben. Der Einfluß einer Reihe von Versuchsbedingungen wie $\mathrm{pH}$, Temperatur und Ionenstärke wurde systematisch untersucht. Durch gezielte Anderung dieser Variablen konnte das "Normalresultat" praktisch beliebig verschoben und damit in einen Bereich gelegt werden, in dem sich die euthyreoten von den hyper- und hypothyreoten Werten am besten abgrenzen lassen. Der entscheidende Einfluß der Versuchsbedingungen auf das Testresultat zeigt u. a., daß mit allen angegebenen Verfahren nicht die tatsächliche relative Verteilung von freiem und gebundenem Trijodthyronin gemessen wird, sondern nur ein von den in vitro-Bedingungen streng abhängiger artifizieller Wert.
\end{abstract}

A simplified gel-filtration method is described for the routine determination of the bound and free I $^{131}$-labelled triiodothyronine in serum. The affects of a number of experimental conditions, like $\mathrm{pH}$, temperature and ionic strength, were systematically investigated. By controlling these variables, the "normal resultt" could be shifted practically as desired. A region could thereby be chosen, in which the values for euthyroidism were separated most markedly from those for hyper- and hypothyroidism. The decisive affect of the experimental conditions on the test results is shown by the fact that all methods give only an artificial value, which depends on the in vitro conditions, and the actual relative distribution of free and bound triodothyronine is never obtained.

Die Schilddrüsenhormone werden nach der Sekretion ins Blut zum weitaus größten Teil an Eiweiße gebunden. Nur etwa $1 \%$ des Thyroxins (, $\left.\mathrm{T}_{4}{ }^{\circ}\right)$ im Blut findet sich in freier, biologisch aktiver Form $(1,2,3)$. Die Bindung folgt dem Massenwirkungsgesetz mit hoher Assoziationskonstante:

$$
\mathrm{T}_{4}+\mathrm{P} \rightleftharpoons \mathrm{T}_{4} \mathrm{P} \quad \mathrm{P}=\text { Protein }
$$

Vorausgesetzt, daß die verschiedenen Bindungsstellen an Serumeiweiß gleich stark sind, gilt in Annäherung (4):

$$
\left[\mathrm{T}_{\mathbf{4}}\right]_{\mathrm{f}}=\mathrm{k} \cdot \frac{\left[\mathrm{T}_{\mathbf{4}}\right]_{\mathrm{t}}}{\left[\mathrm{P}_{\text {ungesättigt } \mathrm{t}}\right]} \quad \begin{aligned}
& \mathrm{f}=\text { frei } \\
& \mathrm{t}=\text { total }
\end{aligned}
$$

Die Schilddrüsenhormone haben eine selektive Affinität für bestimmte Eiweißfraktionen, und zwar für das thyroxinbindende Globulin („TBG“), ein inter $\alpha$ Globulin, für Albumin und thyroxinbindendes Praealbumin (,TBPA"). Die Bindung von Tyroxin ist stärker als die von Trijodthyronin $\left(, \mathrm{T}_{3}{ }^{\mathrm{c}}\right)(5)$. Das Verteilungsmuster der Schilddrüsenhormone im Blut, die physiologische Bedeutùng der einzelnen Vehikelproteine und die physikochemischen Gesetze, die die Bindung bestimmen, sind von zahlreichen Autoren untersucht

Abkürzungen: $T_{4}$ : Thyroxin; $T_{9}$ : Trijodthyronin; $T_{s} 1911$ : mit dem radioaktiven Isotop 1sil markiertes $T_{3}$; TBG: Thyroxin-bindendes Globulin; TBPA: Thyroxin=bindendes Praealbumin; SD: Streuung des Einzelwertes (Standard deviation); SEM: Mittlere Abweichung des Mittelwertes. worden (2,5-10). Die freie Bindungskapazität der Serumproteine hängt vom Funktionszustand der Schilddrüse ab: die Absättigung ist größer bei Hyper-, geringer bei Hypothyreose. Zudem ist wahrscheinlich die Bindungskapazität des TBG bei Hypothyreose gesamthaft erhöht (8). Diese Erkenntnisse können zu diagnostischen Zwecken ausgenützt werden. Wird einem Serum in vitro eine geringe Menge ${ }^{131} \mathrm{I}$-markiertes Trijodthyronin (, $\mathrm{T}_{3}{ }^{131} \mathrm{I}^{\prime \prime}$ ) zugesetzt, so wird der Anteil der eiweißgebundenen Radioaktivität u. a. von der Anzahl der freien Bindungsstellen bestimmt. $\mathrm{Da} \mathrm{T}_{4}$ stärker gebunden wird als $T_{3}$, findet keine nennenswerte Verdrängung statt. Auf dieser Grundlage sind in den letzten Jahren diagnostische Teste in mehreren Spielarten ausgearbeitet worden (11). 1955 berichtete HAMOLSKY über eine Methode, die auf der Inkubation des Gesamtblutes mit $T_{3}{ }^{131} \mathrm{I}$ und Messung des an die Erythrozyten gebundenen Anteils beruhte. Durch Kreuzversuche wurde festgestellt, daß für das Ergebnis das Plasma und nicht die Erythrozyten entscheidend waren (12). Der Test erwies sich als brauchbares Hilfsmittel in der Abklärung von Schilddrüsenerkrankungen $(13,14)$. Andere Autoren verwendeten zur Aufnahme des nicht proteingebundenen $\mathrm{T}_{3}{ }^{131} \mathrm{I}$ anstelle der Erythrozyten Austauschharze $(15,16,17)$ oder die Gelfiltration mit Sephadex (18-21) und erzielten klinisch vergleichbare Resultate. Diese Methoden sind einfacher als das von 